\title{
Primary Mature Cystic Teratoma Mimickıng an Adrenal Mass in an Adult Male Patient
}

\author{
Emrah Okulu, Kemal Ener, Mustafa Aldemir, Evren Isik, Cigdem Irkkan ${ }^{1}$, Onder Kayigil \\ The Urology Clinics of Ankara Ataturk Training and Research Hospital, Ankara, ${ }^{1}$ The Pathology Clinics of Ankara Ataturk Training \\ and Research Hospital, Ankara, Turkey
}

\begin{abstract}
Teratomas are bizarre neoplasms derived from embryonic tissues that are typically found only in the gonadal and sacrococcygeal regions of adults. Primary retroperitoneal teratomas are rare and present challenging management options. We report a case of a unilateral primary retroperitoneal mature cystic teratoma mimicking an adrenal mass in a 54-year-old male patient. Complete resection of the adrenal mass was performed by the flank approach by using the 11th rib resection. Because of the risk of malignancy, follow-up radiographic studies were performed to ensure the oncologic efficacy of resection. The patient has been free of recurrence for longer than 12 months.
\end{abstract}

\section{Keywords: Adrenal neoplasm; Cystic teratoma}

This is an Open Access article distributed under the terms of the Creative Commons Attribution Non-Commercial License (http://creativecommons.org/licenses/by-nc/3.0) which permits unrestricted non-commercial use, distribution, and reproduction in any medium, provided the original work is properly cited.

\author{
Article History: \\ received 21 March, 2012 \\ accepted 6 July, 2012 \\ Corresponding Author: \\ Emrah Okulu \\ Umit Mh. Meksika Cd. 2463. sk. \\ 4/32 Umitkoy, Yenimahalle, \\ Ankara 06810, Turkey \\ TEL: +90312 2354914 \\ FAX: +90312 2912705 \\ E-mail: eokulu@yahoo.com
}

\section{INTRODUCTION}

Primary retroperitoneal teratomas are very rare in adults. They typically occur in this location only in infancy and childhood. Teratomas are tumors that are derived from embryonal tissue and are composed of somatic cell types from two or more germ layers (ectoderm, mesoderm, or endoderm) [1]. A teratoma is considered to be a nonseminomatous germ cell tumor and is typically located in either the sacrococcygeal region or the gonads. Most teratomas in this region (retroperitoneal) are secondary to germ cell tumors of the testes or ovaries. Specifically, in male patients, retroperitoneal germ cell tumors are more likely to have metastasized from the testes than to present as primary tumors [2]. Computed tomography (CT) is very useful for the differential diagnosis of this rare tumor. Here we report a case of unilateral primary mature cystic teratoma of the retroperitoneum mimicking an adrenal mass in an adult male patient.

\section{CASE REPORT}

A 54-year-old previously healthy male patient developed acute, left upper quadrant abdominal and left flank pain. This pain lasted several hours and then resolved spon- taneously. When he was admitted to the hospital, on ultrasound and an abdominal and pelvic CT scan, he was found to have an 8 - $\mathrm{cm} \times 7-\mathrm{cm} \times 6-\mathrm{cm}$ solid and cystic mass in the left (surrenal area) retroperitoneum containing bone and multiple soft tissue densities (Fig. 1). The tumor had calcification and was not well enhanced. There was no evidence of distant metastasis. The tumor was also examined by magnetic resonance imaging (MRI). We also diagnosed that the tumor originated in the left adrenal gland, because the normal adrenal gland could not be recognized by CT or MRI. The patient had hypertension for which he was taking amlodipine $5 \mathrm{mg}$ daily. However, plasma levels of catecholamines, rennin, aldosterone, adrenocorticotropic hormone, and cortisol were within the normal range. Tumor markers, such as serum alpha-fetoprotein, lactate dehydrogenase, carcinoma antigen 125 , neuron-specific enolase, and carbohydrate antigen 19-9, were examined because it was possible that the tumor did not originate from the adrenal gland.

The patient underwent resection of the lesion through a flank incision by 11th rib resection. The retroperitoneal dissection was tedious and difficult, but the mass was excised in its entirety (Fig. 2). The left adrenal gland was normal. The resected retroperitoneal cystic mass measured $8 \mathrm{~cm} \times 7 \mathrm{~cm} \times 6 \mathrm{~cm}$ and weighed $153 \mathrm{~g}$ (Fig. 2). The final 

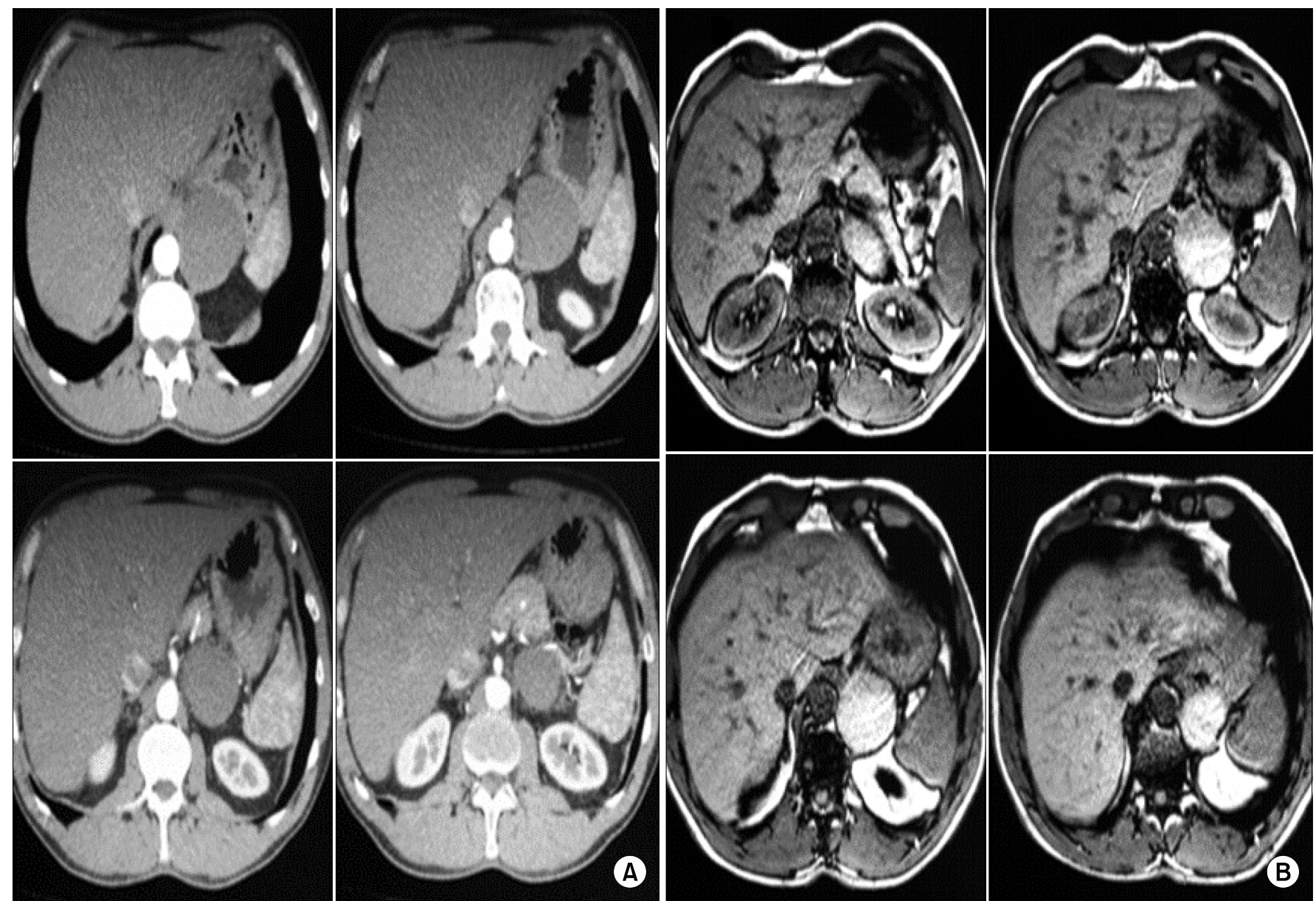

FiG. 1. (A) Abdominal computed tomography demonstrating a left retroperitoneal (surrenal area) mass with solid and cystic components, as well as large and heterogeneous calcifications. (B) Magnetic resonance imaging demonstrating a left retroperitoneal mass with solid and cystic components, as well as large and heterogeneous calcifications.

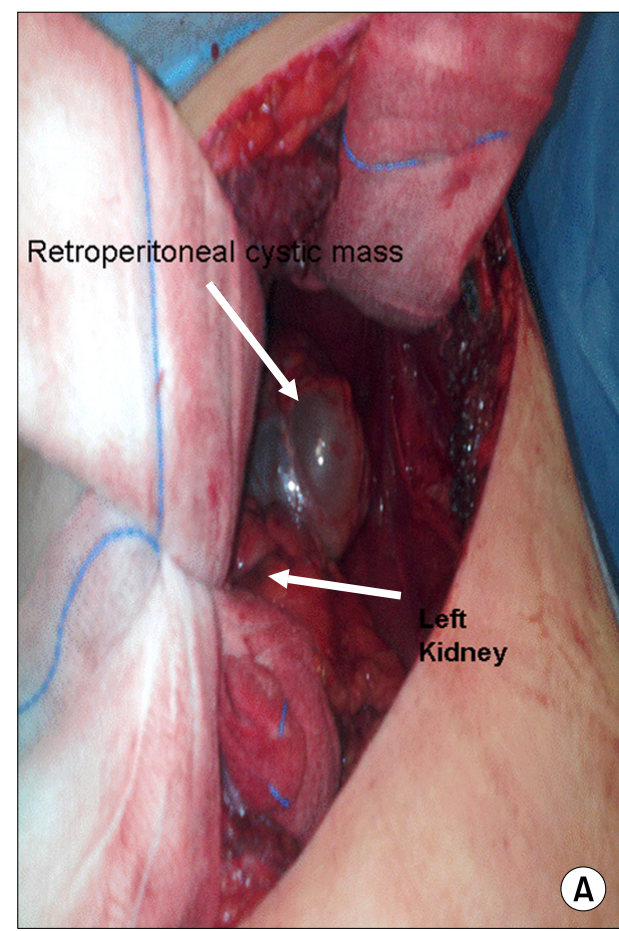

(A)

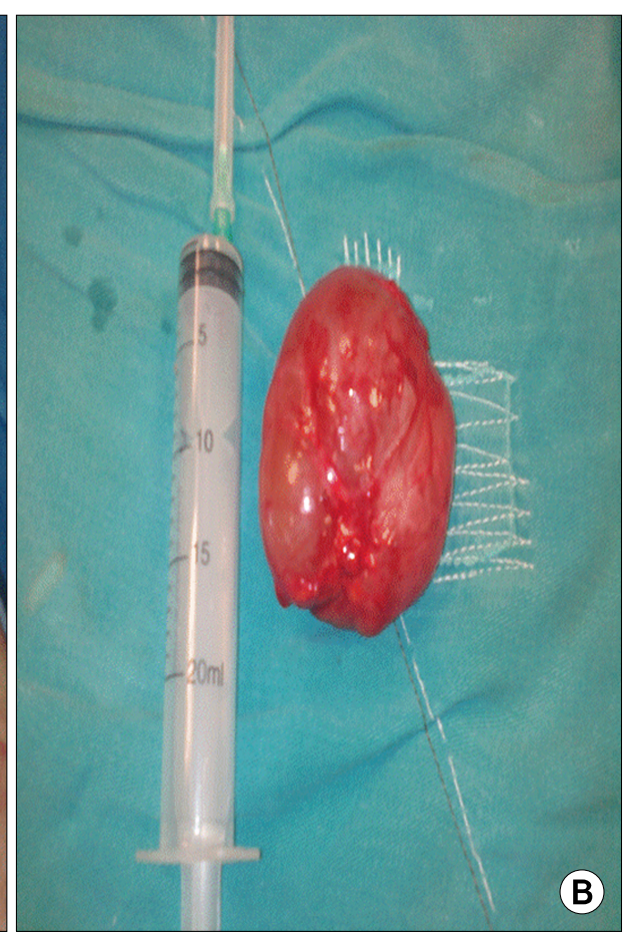

FiG. 2. (A) Left retroperitoneal (surrenal area) cystic mass after flank incision. (B) Gross photograph of the cystic mass. 

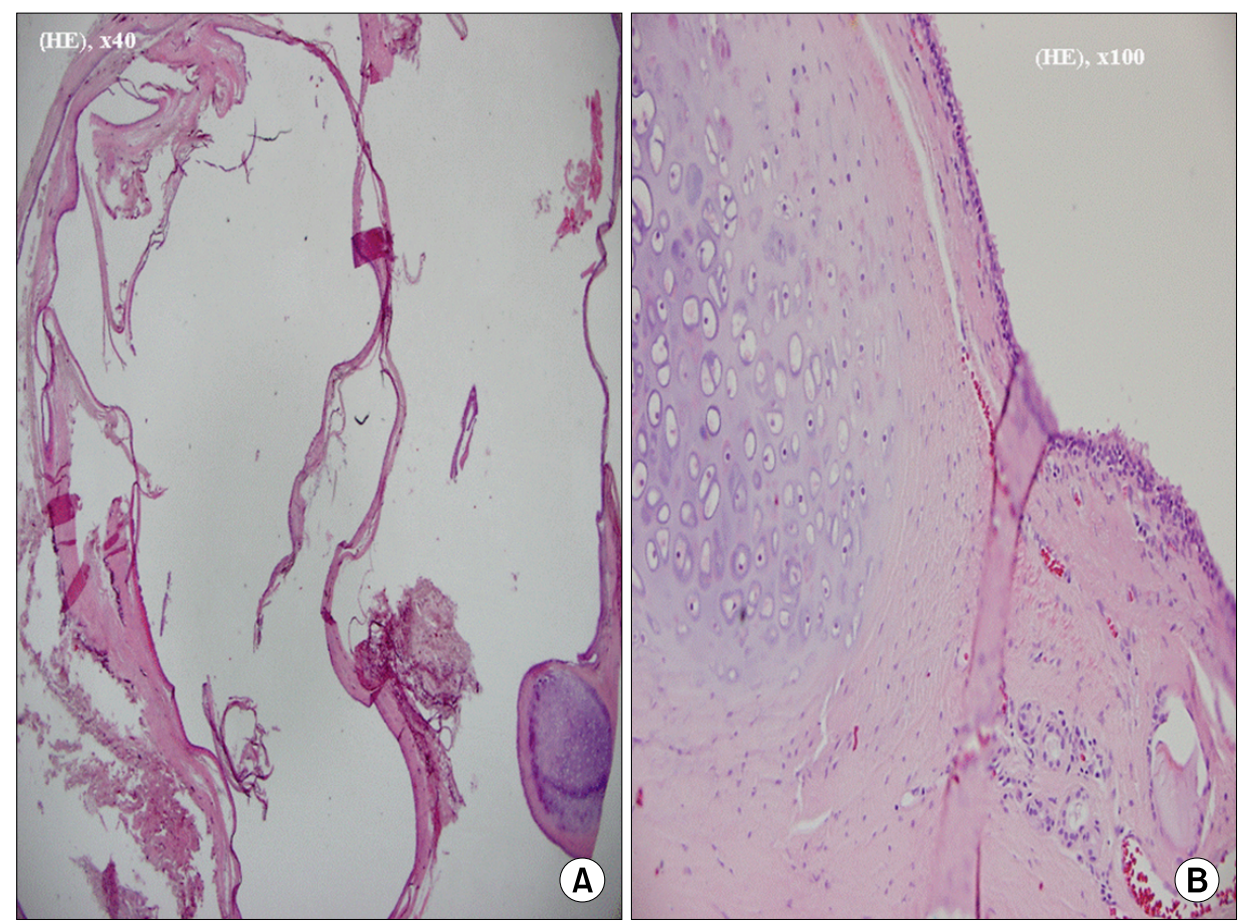

Fig. 3. (A) Histologically thin cyst walls were lined by ciliated columnar epithelial cells inside (H\&E, $\times 40)$. (B) Under the epithelium, hyalin cartilage islands (left) and some seromucinous glands (right) were seen $(\mathrm{H} \& \mathrm{E}, \times 100)$.

pathological evaluation of the tumor was a mature teratoma without malignant components. Macroscopically, the specimen was a $6 \mathrm{~cm} \times 5 \mathrm{~cm} \times 4 \mathrm{~cm}$ fluctuating mass. On incision, cystic material came off and a thinly walled cyst was left. The cyst wall thickness was approximately $1 \mathrm{~mm}$ with only some minor excrescents. Histologically, the cystic cavity was lined by ciliated columnar cells (Fig. 3) and the excrescents were hyalin cartilage islands under this epithelium with some lobules of seromucinous glands (Fig. 3). No immature elements were seen and the diagnosis was a mature cystic teratoma pathologically. Because these tumors usually represent metastasis from other primary sites, additional imaging with CT of the chest and scrotal ultrasonography was performed. No other primary tumor was identified. Therefore, we diagnosed the mass as a primary retroperitoneal teratoma. After 12 months of follow-up, the patient was free of recurrence.

\section{DISCUSSION}

Teratomas are rare congenital neoplasms that develop from more than one and usually all three of the primordial germ cells, which differentiate to form ectodermal, mesodermal, and endodermal tissue elements. During the fourth week of embryologic development, germ cells originating from the yolk sac migrate in the midline of the fetus along the dorsal mesentery from the urogenital ridge to the developing gonads. Some of the cells do not complete the migration and survive in midline locations such as the pineal gland, anterior mediastinum, retroperitoneum, and sacrococcygeal area, where they differentiate into extragonadal teratomas $[2,3]$. Teratomas are classified as one of four variants: (1) mature, when they contain adult or dif- ferentiated tissue; (2) immature, when they comprise predominantly embryonic or undifferentiated tissue; (3) teratoma with malignant transformation; and (4) monodermal, when there is a predominance of tissue arising from one germ cell layer. Mature teratomas occur most often in the ovaries and testes. Extragonadal sites account for $15 \%$ of all teratomas, and the retroperitoneum is the least common location [4].

The diagnosis of a retroperitoneal teratoma can often be made on the basis of radiologic imaging. Retroperitoneal teratomas can be predominantly cystic or completely solid in appearance. A CT scan or MRI can identify various components of these tumors, including bone, soft-tissue density structures, adipose tissue, and sebaceous and serous-type fluids. These imaging studies can also display the precise location, morphology, and adjacent structures of the tumor, which provide better preoperative planning and increased likelihood of complete removal of the tumor with less iatrogenic damage [5].

A primary retroperitoneal mature cystic teratoma in a 54-year-old man is a rare phenomenon. Most retroperitoneal teratomas in adults represent metastases from a primary gonadal tumor [6]. Our patient had a retroperitoneal mature cystic teratoma that was not derived from any specific organ. Radiographic evaluation did not confirm the origin or the nature of the retroperitoneal mass.

Germ cell tumors in the retroperitoneum usually occur in pediatric populations [7]. Retroperitoneal mature cystic teratomas are characterized by a bimodal peak in incidence, occurring in the first 6 months of life and in early adulthood [2]. Primary retroperitoneal teratomas in adults are uncommon, with only 32 cases reported between 1937 
and 1987 [2]. In adults, documented cases of retroperitoneal teratomas are often secondary sites of tumor genesis. Primary tumors metastasizing to this region have been identified in the breasts, lungs, and gonads. Specifically, in male patients, retroperitoneal germ cell tumors are more likely to have metastasized from the testes than to present as primary tumors [2]. Primary retroperitoneal teratomas in adults are usually found in the upper portion of the left kidney [2]. In the case of a retroperitoneal tumor, germ cell tumors should be considered and tumor markers examined before surgery [8]. The malignancy rate of $25.8 \%$ in adults is significantly higher than the $6.8 \%$ rate documented in children [2]. Regardless of the benign histological nature of mature teratomas, close follow-up is recommended because the incidence of malignant transformation is approximately $3 \%$ to $6 \%$. In the present case, the patient was free of recurrence after 12 months of follow-up.

In conclusion, primary retroperitoneal teratoma is a rare entity in adults. Although usually asymptomatic, large neoplasms can cause abdominal and flank pain. Preoperatively, the diagnosis can be established by its characteristic appearance on CT. Although retroperitoneal teratomas can be radiologically recognized, it is important to note that masses in the suprarenal region are likely to be confused with suprarenal masses, as in our case. The definitive primary treatment of retroperitoneal teratomas is surgical resection.

\section{CONFLICTS OF INTEREST}

The authors have nothing to disclose.

\section{REFERENCES}

1. Taori K, Rathod J, Deshmukh A, Sheorain VS, Jawale R, Sanyal $\mathrm{R}$, et al. Primary extragonadal retroperitoneal teratoma in an adult. Br J Radiol 2006;79:e120-2.

2. Gatcombe HG, Assikis V, Kooby D, Johnstone PA. Primary retroperitoneal teratomas: a review of the literature. J Surg Oncol 2004; 86:107-13.

3. Scott AL, Abbassi-Ghadi N, Archer CM, Swamy R, Gupta S. Neuroendocrine carcinoma arising within a retroperitoneal mature teratoma. Ann R Coll Surg Engl 2010;92:W5-8.

4. Bedri S, Erfanian K, Schwaitzberg S, Tischler AS. Mature cystic teratoma involving adrenal gland. Endocr Pathol 2002;13:59-64.

5. Liu H, Li W, Yang W, Qi Y. Giant retroperitoneal teratoma in an adult. Am J Surg 2007;193:736-7.

6. Sato F, Mimata H, Mori K. Primary retroperitoneal mature cystic teratoma presenting as an adrenal tumor in an adult. Int J Urol 2010;17:817.

7. Eyssartier E, Harper L, Michel JL, Riviere JP, Vanderbecken S, De Napoli-Cocci S. Rapidly growing mature retroperitoneal teratomas. J Pediatr Hematol Oncol 2009;31:705-6.

8. Rais-Bahrami S, Varkarakis IM, Lujan G, Jarrett TW. Primary retroperitoneal teratoma presenting as an adrenal tumor in an adult. Urology 2007;69:185.e1-2. 